\title{
Melting of 2D liquid crystal colloidal structure
}

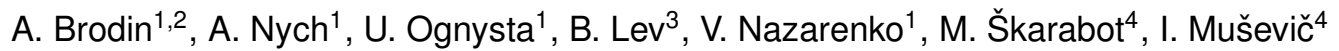 \\ ${ }^{1}$ Institute of Physics NAS Ukraine, Kyiv, Ukraine \\ 2 National Technical University of Ukraine "Kyiv Polytechnic Institute", Kyiv, Ukraine \\ 3 Bogolyubov Institute for Theoretical Physics NAS Ukraine, Kyiv, Ukraine \\ 4 J. Stefan Institute, Jamova 39, 1000 Ljubljana, Slovenia
}

Received January 4, 2010, in final form May 25, 2010

\begin{abstract}
Using video microscopy, we investigated melting of a two-dimensional colloidal system, formed by glycerol droplets at the free surface of a nematic liquid crystalline layer. Analyzing different structure correlation functions, we conclude that melting occurs through an intermediate hexatic phase, as predicted by the KosterlitzThouless-Halperin-Nelson-Young(KTHNY) theory. However, the temperature range of the intermediate phase is rather narrow, $\lesssim 1^{\circ} \mathrm{C}$, and the characteristic critical power law decays of the correlation functions are not fully developed. We conclude that the melting of our 2D systems qualitatively occurs according to KTHNY, although quantitative details of the transition scenario may partly depend on the details of interparticle interaction.
\end{abstract}

Key words: liquid crystal, $2 D$ colloidal structure, phase transitions in $2 D$ structures

PACS: $61.30 .-v, 64.70 . p v, 82.70 . D d$

\section{Introduction}

Two-dimensional (2D) ordering and the formation of periodic 2D crystal-like structure, although less familiar than 3D crystallization, is still widespread enough and can even be observed in the cup of morning coffee as Bénard convection cells [1]. In recent decades, 2D systems and their order-disorder transition has received considerable attention in computational and experimental physics. Laboratory $2 \mathrm{D}$ systems are usually formed by colloidal particles that are large enough to be individually observable by optical means, so that the structure and even individual particle trajectories can be monitored by optical microscopy, thus making the complete structural and dynamical data readily experimentally accessible [2]. For instance, charged dust particles in "dusty plasma" readily form the so-called Wiegner or Coulomb crystal, whose melting transition can be experimentally investigated by optical means [3].

2D lattices are in many respects similar to and considered as convenient models for 3D systems, although it has been recognized that the analogy is only partial and that, in certain respects, 2D order has characteristics that are unique to its dimensionality [4]. In particular, it has been shown that $1 \mathrm{D}$ and $2 \mathrm{D}$ crystalline lattices would be thermally unstable, and thus spontaneous crystalline order should not exist in one or two dimensions [5, 6], which is sometimes quoted as PeierlsMermin theorem [4]. A more familiar consequence of this property is the absence of true long-range positional order in the two-dimensional crystal, so that its positional correlation function decays algebraically to zero with distance, in contrast to the 3D case where a finite value is attained. This led Kosterlitz and Thouless to suggest [7] that the nature of the melting transition in 2D is different from 3D and to formulate a theory of melting as a continuous transition mediated by the dissociation of dislocation pairs. The problem of $2 \mathrm{D}$ melting was subsequently worked out in greater detail by Halperin, Nelson, and Young [8, 9], with the resulting microscopic theory now commonly referred to as Kosterlitz-Thouless-Halperin-Nelson-Young (KTHNY) theory. It has to be noted that no comparable microscopic theory exists in 3D, which again sets the 2D case apart. Qualitatively, KTHNY predicts a two-stage 2D melting that occurs from the hexagonal (equilateral 
triangular) through an intermediate "hexatic" phase, in which the positional and bond-orientational correlation functions exhibit characteristic algebraic decays, and only via a second transition does the system become an ordinary fluid where all correlation functions decay exponentially. Since the formulation of KTHNY theory, the 2D melting transition has been a matter of continuous debate. Several computer simulations and experiments have been performed (for a review, see [1012]), although the results were not always consistent. Overall, it has to be noted that KTHNY only describes how a 2D crystal could melt, so that other scenarios cannot be excluded. For instance, it appears that the KTHNY transitions can be preempted by other processes leading to a single first order transition as in 3D [13]. On the other hand, experiments on paramagnetic colloidal particles, whose dipolar interaction could be conveniently controlled by magnetic field, concluded a perfect quantitative agreement with the two-stage KTHNY scenario [14, 15]. Similar conclusion followed from a recent work on charged millimeter-sized steel balls [20]. Yet another experimental work on uncharged polymer microspheres [16], while confirming the existence of hexatic phase, concluded that the liquid-to-hexatic and hexatic-to-solid transitions are strongly first order transitions. A work on charged colloids [17], while confirming the existence of an intermediate phase, concluded that this phase was different from the KTHNY expectations. Several works on dusty plasmas (e. g., [3, 18, 19]) appear to point towards a grain-boundary-induced rather than KTHNY melting scenario. Overall, it appears that the KTHNY scenario may not be truly universal but rather depends on the specific properties of the systems, predominantly related to the details of interparticle interaction, so that further investigation of systems with different interactions is highly motivated. Recently, there has been discovered a new class of self-ordering 2D colloidal systems, formed by colloidal particles (droplets) at the free surface of a nematic liquid crystal (NLC) [2123]. These colloids, which interact via liquid crystal mediated elastic forces, readily form hexagonal crystals that exhibit melting transition at temperatures in the range $T_{\text {melting }}=26 \div 28^{\circ} \mathrm{C}$, which are relatively far from the temperature of the nematic-isotropic liquid transition, $T_{\mathrm{NI}}=36^{\circ} \mathrm{C}$. Such a colloidal system allows for observation of the $2 \mathrm{D}$ melting phenomena over the large area with a constant colloidal fraction, size of colloids, and interaction between the particles, as the main effect of the temperature on the system occurs only through the magnitude of thermal fluctuations of the colloidal particles. In the present contribution, we perform an analysis of the melting transition in these systems in an attempt to test the applicability of KTHNY and its predictions to this novel class of $2 \mathrm{D}$ lattices.

\section{Experiment}

In our experiments we have used hexagonal array of glycerol droplets trapped at the NLCair interface and stabilized by the long range elastic-capillary interactions [21, 23]. A cleaned glass plate of $2 \times 2 \mathrm{~cm}$ size was covered with the polymer (Elvamide 8061) film and rubbed to ensure good planar alignment of NLC 5CB (4-n-pentyl-4-cyanobiphenyl, Merck). A layer of 5CB (clearing temperature $T_{\mathrm{NI}}=36^{\circ} \mathrm{C}$ ) of $40 \div 60 \mu \mathrm{m}$ thickness was deposited directly onto the substrate and allowed to equilibrate for 10 minutes. Then the substrate with 5CB film was held upside down above a petri dish filled with glycerol. The petri dish was heated up to $120^{\circ} \mathrm{C}$ to facilitate transfer of glycerol onto the NLC layer. During this process, the glycerol evaporates from the petri dish and condenses on the NLC layer forming almost uniform size droplets (see figure 1] (a)). By adjusting deposition time and distance between the glycerol surface and substrate, the amount of condensed glycerol can be altered and therefore the number and size of the glycerol droplets can be effectively controlled. The procedure generally yields $2 \mathrm{D}$ crystalline hexagonal lattice. After the deposition, the substrate with NLC film was placed onto a microscope hot stage (MK1, Instec Inc.) for observation and temperature control. Figure 1 (b) shows an optical microscopy image of such hexagonal structure at the free surface of the NLC layer. From the optical images (blurred by diffraction) of the droplets we estimated their size to be $2 R \simeq 1.0 \pm 0.2 \mu \mathrm{m}$, and the lattice constant $a \simeq 1.9 \pm 0.1 \mu \mathrm{m}$. This estimation is further justified by an observation that the glycerol droplets exhibit Brownian motion with considerable magnitude, suggesting that their surface-to-surface separation is $\approx 0.5 a$, so that the structure is not densely packed [23]. 
a)

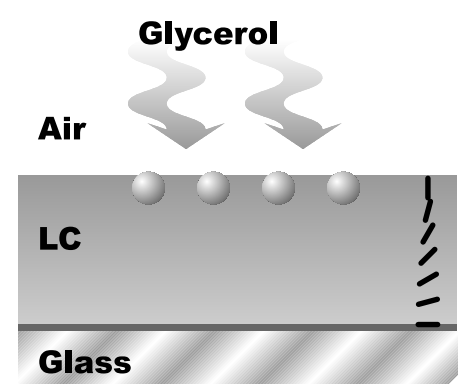

b)

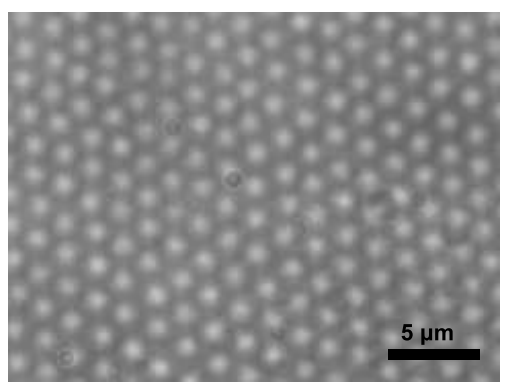

Figure 1. (a) Schematic representation of the preparation procedure of $2 \mathrm{D}$ colloidal structure formed by glycerol droplets trapped at the free surface of a nematic liquid crystal film at $26^{\circ} \mathrm{C}$. Black lines at the right side show the orientation of LC molecules (NLC director) inside the LC layer, which changes from planar at the bottom to homeotropic at the top surface. (b) Microscopic image of the 2D colloidal lattice, formed by glycerol droplets at the LC-air interface. The structure on the snapshot is not ideal hexagonal due to considerable Brownian motion of the droplets.

Digital images of the 2D structures, taken at different temperatures, were first analyzed in terms of the particle positions (coordinates) by using custom-written MatLab programs according to computerized videomicroscopy algorithms [24]. From the so-obtained data of $(x, y)$ coordinates of the particles' centers, we then computed various structural correlation functions, discussed below. In order to find the structural "bonds" between the nearest neighbors, we performed Delaunay triangulation procedure on the sets of particle positions, obtained from recorded video-frames. The resulting structural data in terms of bond positions, lengths, and orientations, were used for visualization of structural changes during melting (see figure 2), and for further statistical analyses, such as determination of the number of nearest neighbours of each particle, and computing bondorientational correlation functions.

\section{Results and discussion}

Our 2D hexagonal crystals, figure 1, exhibit "softening" and melting well below the nematicto-isotropic transition of $5 \mathrm{CB}\left(\simeq 36^{\circ} \mathrm{C}\right.$ in our case). In order to visualize the structural changes upon softening and melting, we show in figure 2 snapshots of the structure at four representative temperatures, presented as wire frames of interparticle bonds.
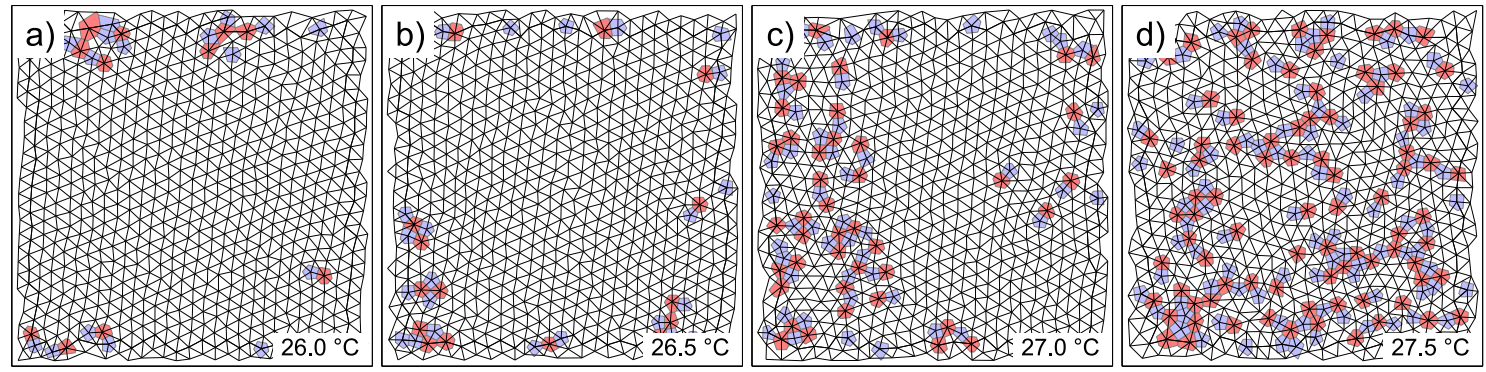

Figure 2. Structural changes of the ordered phase of colloidal droplets with changing the temperature. Positions of the glycerol droplets were digitized and Delaunay triangulation mesh was calculated to represent lattices at different temperatures. Lattice sites with number of neighbors greater or smaller than six are highlighted with red and blue color respectively. Increasing number of lattice defects as well as disappearance of the long-range ordering indicates melting of the colloidal crystal.

From visual inspection of figure 2 the close to perfect hexagonal structure observed at $26^{\circ} \mathrm{C}$ is almost completely lost by $27.5^{\circ} \mathrm{C}$, although the structural changes are obviously gradual, i. e. the 
loss of crystalline order occurs through continuous increase of the number of defects, as seen at $27^{\circ} \mathrm{C}$ and $27.5^{\circ} \mathrm{C}$, rather than through an abrupt transition at any particular temperature. Thus, melting of our colloidal system is a continuous rather than first-order transition, and therefore it qualitatively corresponds to the KTHNY scenario.

From the particular examples of figure 2 it may appear that the creation of structural defects with increasing the temperature is not uniform, but rather occurs through diffusion of the defects from the outer parts of the structure towards the center, so that the structural disordering and concomitant melting are rather grain-boundary-induced, similarly to the results of, e. g., [3, 18, 19]. This would mean a grain-boundary-induced transition [13], which is different from the KTHNY scenario. As far as we cannot exclude the occurrence of grain-boundary-induced melting in some of our colloidal structures, we note that in most cases the structural changes were rather uniform, qualitatively in accord with KTHNY.

We now proceed to quantitative analysis of the structural changes upon softening and melting, and compute the radial distribution function $g(|\mathbf{r}|)$ of the particles according to

$$
g(|\mathbf{r}|)=\frac{1}{N}\left\langle\sum_{j \neq k}^{N} \delta\left(\mathbf{r}+\mathbf{r}_{j}-\mathbf{r}_{k}\right)\right\rangle,
$$

where $N$ is the number of particles and $\langle\ldots\rangle$ denotes ensemble average over a series of structures, photographed at the same temperature. Figure 3 shows $g(r)$ at several selected temperatures. The peaks at $27.5^{\circ} \mathrm{C}$ are smeared out in comparison with $26^{\circ} \mathrm{C}$ - in particular, the characteristic splitting of the second peak that corresponds to the second $(\sqrt{3} a)$ and third $(2 a)$ neighbor distances in the second coordination shell is no longer recognizable. The disappearance of the splitting is obviously associated with crystal melting, although it is not possible to use it as a quantitative indicator, since the peak broadening occurs not only because of structural changes but also due to increased fluctuations of particle positions at higher temperatures. The first peak, whose area gives the coordination number, appears to somewhat decrease in magnitude, which can be attributed to the growing number of structural defects with increasing temperature. At large distances, the peaks broaden, merge with one another, and become lost in the continuum background $g(r) \rightarrow 1$. Overall, it does not appear possible to conclude on the details of structural changes from the pair correlation function. Indeed, it is well known that the pair correlation function cannot sensitively reveal the presence or absence of long-range order, the liquid $g(r)$ being virtually indistinguishable from the one of a "hot solid", whose atoms are displaced from the equilibrium lattice sites due to thermal motion [4].

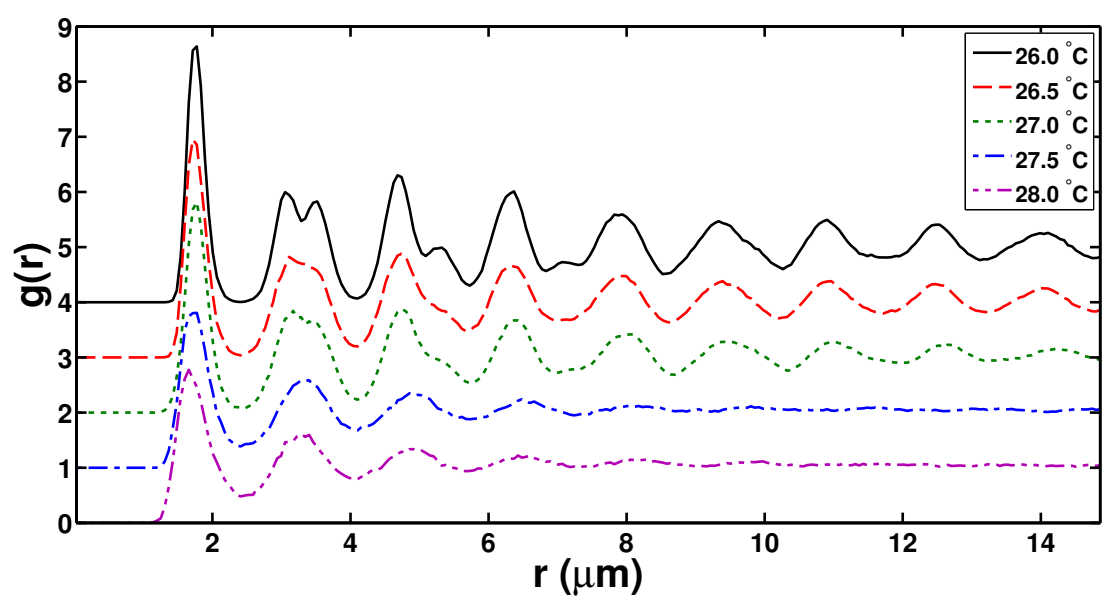

Figure 3. Radial distribution function $g(r)$ of the $2 \mathrm{D}$ colloidal lattice at indicated temperatures. Note that the splitting of the second peak, clearly visible at $26^{\circ} \mathrm{C}$, disappears at higher temperatures, indicating the loss of hexagonal order. Plots are evenly shifted vertically for clarity. 
Next, we analyze the structure factor $S(\mathbf{q})$

$$
S(\mathbf{q})=\frac{1}{N} \sum_{j, k=1}^{N} \mathrm{e}^{-\mathrm{iq} \cdot\left(\mathbf{r}_{j}-\mathbf{r}_{k}\right)},
$$

where $N$ is the number of particles and $\mathbf{r}_{j}, \mathbf{r}_{k}$ their position vectors. For a perfect $3 \mathrm{D}$ crystal with its infinite Bragg planes, $S(\mathbf{q})$ consists simply of an array of delta functions at the points of the reciprocal lattice $\mathbf{q}=\mathbf{G}$, superimposed on a background that is related to the thermal fluctuations of the atoms about their equilibrium positions and that is expressed through the Debye-Waller factor. These Bragg peaks remain infinitely sharp at any temperature, as long as the crystal has not melted, since the atoms occupy, on average, equilibrium lattice sites (long range order). In 2D, as mentioned, the long range order is not expected to occur, and thus the infinite "Bragg lines" do not exist, so that the peaks in $S(\mathbf{q})$ are progressively smeared out with increasing $q$. Figure 4 shows $S(\mathbf{q})$ for a set of about 700 particles.
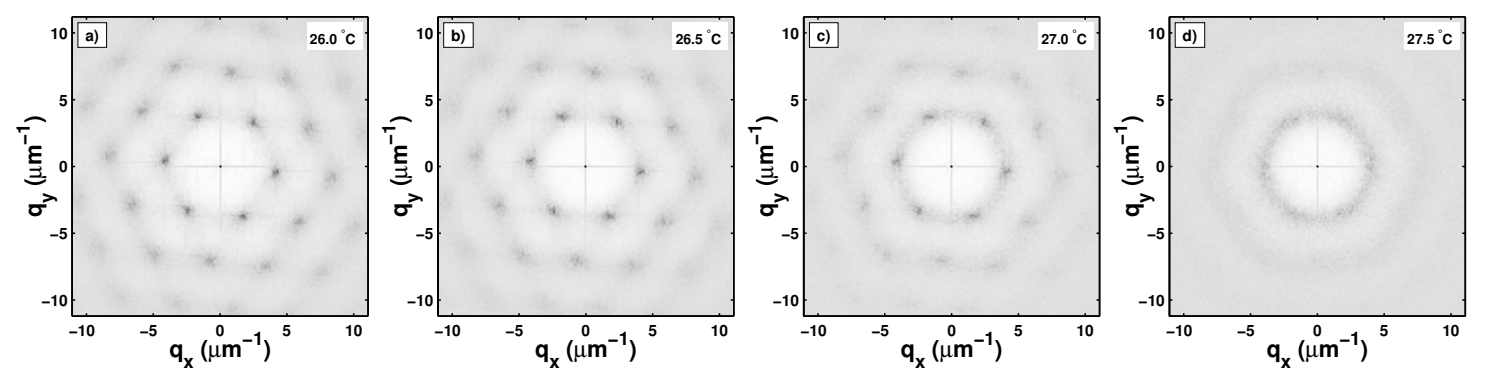

Figure 4. Static structure factor (interference function) $S(\mathbf{q})$ of a system of about 700 particles at selected temperatures. With increase of temperature high-order peaks vanish while six firstorder peaks are blurred into ring, which clearly indicates loss of both positional and angular ordering. Darker shading corresponds to higher magnitude of $S(\mathbf{q})$.

The "diffraction peaks", seen in figure 4 (a), are indeed rather broad and have low intensity, only about a few percent compared to the central peak. Furthermore, the peaks at $q \gtrsim 5 \mu \mathrm{m}^{-1}$ beyond the first Brillouin zone are only barely distinguishable, as really expected in the case of only a relatively short translational order. It is also seen that the lattice is not exactly hexagonal but is somewhat deformed.

When the temperature of the system increases, the first-order peaks of $S(\mathbf{q})$ are smeared out, and by $27.5^{\circ} \mathrm{C}$ almost completely disappear, transforming into a nearly isotropic ring (see figure 4 (d)). Higher-order peaks vanish already at $27^{\circ} \mathrm{C}$, see figure 4 (c). Such changes of $S(\mathbf{q})$ indicate that our system melts at about $27^{\circ} \mathrm{C}$.

It is instructive to compare characteristic changes of the $2 \mathrm{D}$ structure factor on melting with those in the 3D case. One of the well-known criteria of freezing of a monatomic 3D liquid asserts that the amplitude $S\left(q_{0}\right)$ of the main peak of the liquid structure factor reaches a characteristic value of 2.85 at freezing [26], which is known as Hansen-Verlet criterion [12]. Furthermore, the amplitude $S\left(q_{0}\right)$ can be considered as an order parameter in a first-principles theory of freezing 27]. Meanwhile, the corresponding amplitude of the main structure factor peak in $2 \mathrm{D}$ reaches significantly higher values than 2.85 [27 29]. Otherwise, the general applicability of the HansenVerlet criterion in 2D is not clear, nor is the corresponding characteristic value $S\left(q_{0}\right)$. The orderparameter theory of Ramakrishnan and Yussouff [27] suggests the critical value $S\left(q_{0}\right) \approx 7$ in $2 \mathrm{D}$, whereas molecular dynamics simulations rather indicate the value of 5.5. Empirical evidence seems to suggests $\approx 4[12$.

To investigate the temperature behavior of $S\left(q_{0}\right)$, we computed $S(q)$, which is the angular average of the structure factor $S(\mathbf{q})$, from the radial distribution functions $g(r)$ of figure 3 through the (two-dimensional) Fourier transform [4]

$$
S(q)=1+\rho \int[g(r)-1] \mathrm{e}^{-\mathrm{iq} \cdot \mathbf{r}} \mathrm{d} \mathbf{r},
$$


where $\rho$ is the number density. Performing the angular integration in equation (3) leads to the following expression for $S(q)$ through the Hankel transform,

$$
S(q)=1+2 \pi \rho \int_{0}^{\infty}[g(r)-1] J_{0}(q r) r \mathrm{~d} r,
$$

where $J_{0}(q r)$ is the zero-order Bessel function of the first kind.

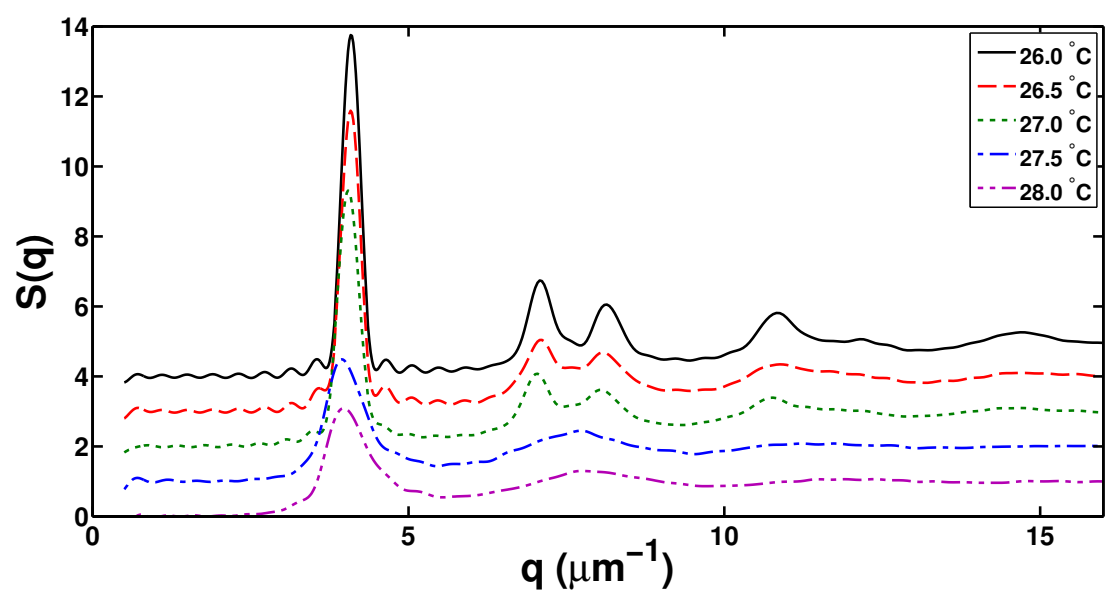

Figure 5. Angle-averaged structure factor $S(q)$ at indicated temperatures (traces for temperatures below $28.0{ }^{\circ} \mathrm{C}$ are evenly shifted vertically).

In figure 5 we present temperature-dependent structure factors $S(q)$ of our systems, numerically computed from the radial distribution functions $g(r)$ of figure 3 with the help of equation (4). It is immediately obvious from the figure that the structure factors, and thus the structure, exhibit a dramatic, almost abrupt change upon the temperature change from $27.0^{\circ} \mathrm{C}$ to $27.5^{\circ} \mathrm{C}$. Firstly, the characteristic splitting of the second peak, clearly seen at $T \leqslant 27.0^{\circ} \mathrm{C}$, disappears above that temperature, and secondly, the amplitude of the first peak decreases almost abruptly from $\gtrsim 7$ to $\approx 3$. The splitting of the second peak of $S(q)$, analogous to the corresponding splitting in $\mathrm{g}(\mathrm{r})$ discussed above, is evidently much more sensitive to the presence of hexagonal order than the second peak of $g(r)$. From its behavior alone one concludes that our 2D structure melts somewhere between $27.0^{\circ} \mathrm{C}$ and $27.5^{\circ} \mathrm{C}$. The concomitant drop of the first peak amplitude also appears to be a sensitive indicator of melting, with the typical amplitude in the molten state of $\sim 3$. The highest amplitude, detected at $27.5^{\circ} \mathrm{C}$ just prior to freezing, amounts to 3.5 , which is rather close to the empirical value of $\approx 4[12]$, but significantly less than the theoretical value of $\sim 7[27]$.

Next we turn to analyzing other correlation functions that are more sensitive to the structural order than the pair correlation function, and for which specific behavior is expected within the KTHNY theory. Firstly, in table 1 we summarize the expected translational and orientational order in different phases, according to the KTHNY theory.

Truly long range order is only expected for bond orientations in the crystalline phase, whereas translational correlation is then only quasi-long range, so that the corresponding correlation function $g_{\mathrm{G}}(r)$ (defined later in equation equation (5)) decays algebraically with distance. In the intermediate hexatic phase, bond-orientational correlation function $g_{6}(r)$ (see later equation (6) for definition) acquires critical algebraic decay. In all cases of short range order, the corresponding correlation functions decay exponentially with distance. Specifically, in the solid phase one expects $g_{\mathrm{G}}(r) \propto r^{-\eta}$ with the exponent $\eta$ in the range $1 / 4 \leqslant \eta\left(T_{\mathrm{m}}\right) \leqslant 1 / 3$ at the "melting" temperature $T_{\mathrm{m}}$, where transition to the hexatic phase starts to occur. Up to a higher temperature $T_{\mathrm{i}}$, at which complete isotropization occurs, the system remains in the hexatic phase with $g_{6}(r) \propto r^{-} \eta_{6}$ with the exponent that assumes the value of $1 / 4$ exactly at $T_{\mathrm{i}}$, i. e. $\eta_{6}\left(T_{\mathrm{i}}\right)=1 / 4$. Thus, one expects 

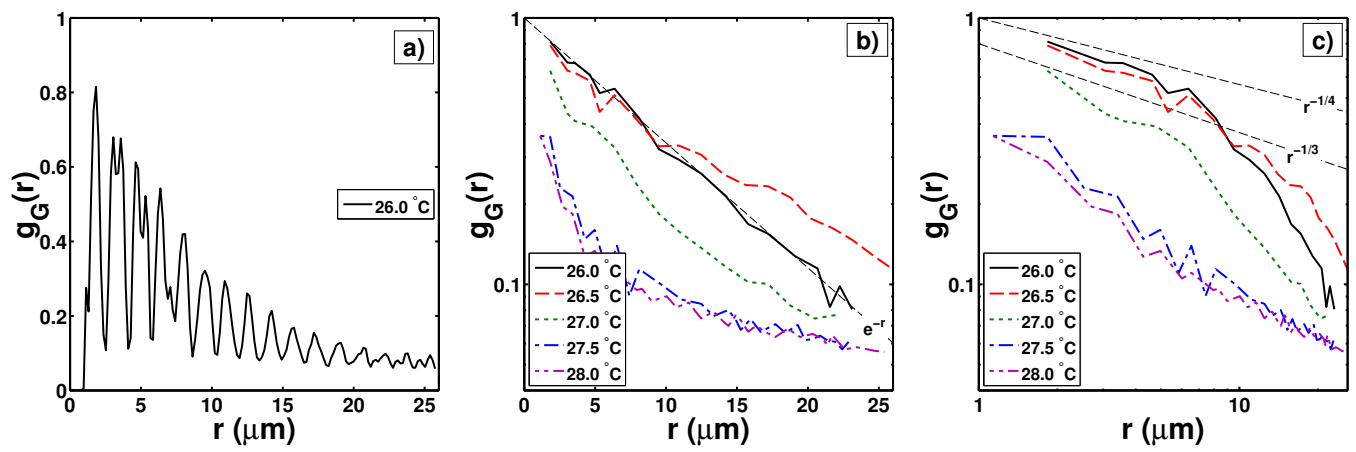

Figure 6. (a) Translational (positional) correlation function $g_{\mathrm{G}}(r)$ at $26^{\circ} \mathrm{C}$. (b), (c) Smoothed $g_{\mathrm{G}}(r)$ (envelopes of the oscillating functions) at selected temperatures, on the semi-log and loglog scale, respectively. Dashed lines represent characteristic exponential and power-law decays.

Table 1. Translational and orientational order in different phases, according to the KTHNY theory.

\begin{tabular}{|c|c|c|c|}
\hline & solid & hexatic & liquid \\
\hline $\begin{array}{c}\text { Translational } \\
\text { order }\end{array}$ & $\begin{array}{l}\text { quasi- } \\
\text { long- } \\
\text { range }\end{array}$ & $\begin{array}{l}\text { short- } \\
\text { range }\end{array}$ & $\begin{array}{l}\text { short- } \\
\text { range }\end{array}$ \\
\hline $\begin{array}{l}\text { Bond- } \\
\text { angle } \\
\text { order }\end{array}$ & $\begin{array}{l}\text { long- } \\
\text { range }\end{array}$ & $\begin{array}{l}\text { quasi- } \\
\text { long- } \\
\text { range }\end{array}$ & $\begin{array}{l}\text { short- } \\
\text { range }\end{array}$ \\
\hline
\end{tabular}

characteristic power law decays of the correlation functions at the characteristic temperatures $T_{\mathrm{m}}$ and $T_{\mathrm{i}}$.

We first consider the translational (positional) correlation function $g_{\mathrm{G}}(r)$, defined as follows [8]:

$$
\begin{aligned}
g_{\mathrm{G}}\left(\left|\mathbf{r}-\mathbf{r}^{\prime}\right|\right) & =\left\langle\exp \left[\mathrm{i} \mathbf{G} \cdot\left(\mathbf{h}(\mathbf{r})-\mathbf{h}^{\prime}\left(\mathbf{r}^{\prime}\right)\right)\right]\right\rangle \\
& =\left\langle\exp \left[\mathrm{i} \mathbf{G} \cdot\left(\mathbf{r}-\mathbf{r}^{\prime}\right)\right]\right\rangle
\end{aligned}
$$

where $\mathbf{G}$ denotes a reciprocal lattice vector, $\mathbf{h}(\mathbf{r})=\mathbf{r}-\mathbf{R}$ is the displacement of the particle at $\mathbf{r}$ with respect to the ideal lattice site $\mathbf{R}$, and the last equality follows from the fact that $\exp \left[\mathrm{i} \mathbf{G} \cdot\left(\mathbf{R}^{\prime}-\mathbf{R}\right)\right]=1$ for any $\mathbf{R}^{\prime}, \mathbf{R}$. The translational correlation function $g_{\mathrm{G}}(r)$ measures the decay of translational order with distance. In a $3 \mathrm{D}$ solid, it would decay to a finite value at large $r$ (long range order), whereas in the $2 \mathrm{D}$ case we expect it to decay algebraically to zero.

Figure 6 (a) shows the translational correlation function $g_{\mathrm{G}}(r)$ at $26^{\circ} \mathrm{C}$, computed with the reciprocal lattice vectors, obtained from figure 4. The correlation function exhibits strong oscillation, reminiscent of the corresponding oscillation of the radial distribution function $g(r)$ in figure 3 and reflecting the local order. We are mainly interested in the loss of correlation with distance which is reflected in the behavior of the upper envelope function, rather than fast oscillations due to the local order. We therefore extract only the peak maxima from the oscillating $g_{\mathrm{G}}(r)$, computed at different temperatures, and plot those envelopes in figure 6 (b) and (c) on a semi-log and log-log scale, respectively.

Surprisingly, inspecting figure 6 (b) it appears that at the lowest temperatures $26^{\circ} \mathrm{C}$ and $26.5^{\circ} \mathrm{C}$, which correspond to a solid hexagonal structure, the correlation decay is close to exponential, see dashed line in figure 6 (b), with a correlation length $\xi=9.3 \mu \mathrm{m}=5.3 a$. At higher temperatures the correlation length decreases sharply, although the decay, rather than becoming/remaining exponential, appears to turn algebraic, see figure 6 (c). We cautiously note that such an "anomalous" behavior, while probably being genuine for our systems, may also be an artifact due to the limited 
structure size and numerical inaccuracies. One of the possible sources of such inaccuracies is in the determination of the reciprocal lattice vector $\mathbf{G}$, which enters equation (5). Obviously, the reciprocal vector cannot be exactly defined - in fact, none exists in the true sense, due to the lack of long range order.

At distances shorter than the correlation length $\xi$, the decay of $g_{\mathrm{G}}(r)$ at $T \leqslant 26.5^{\circ} \mathrm{C}$ can be approximated with a power law, whose exponent $\eta$ is close to $1 / 3$ or $1 / 4$, see the $\log$-log plot of figure 6 (c). At $27^{\circ} \mathrm{C}$ the decay is already somewhat faster than $r^{-1 / 3}$, whereas at $T \geqslant 27.5^{\circ} \mathrm{C}$, when the structure must have melted according to the preceding analysis, the decay, albeit not truly exponential, is clearly much faster than $r^{-1 / 3}$. Thus, it appears that the hexatic-like phase only occurs in the narrow temperature range of $\approx 26.5^{\circ} \mathrm{C}$ to $27^{\circ} \mathrm{C}$, i. e. over a mere $1^{\circ} \mathrm{C}$ or less.

Now we turn the attention to the bond-orientational correlation function $g_{6}(r)$, defined as follows [10]:

$$
g_{6}\left(\left|\mathbf{r}_{b}-\mathbf{r}_{b}^{\prime}\right|\right)=\left\langle\exp \left[6 \mathrm{i} \theta\left(\mathbf{r}_{b}, \mathbf{r}_{b}^{\prime}\right)\right]\right\rangle,
$$

where $\theta\left(\mathbf{r}_{b}, \mathbf{r}_{b}^{\prime}\right)$ is the angle between the bonds at $\mathbf{r}_{b}$ and $\mathbf{r}_{b}^{\prime}$. The bond-orientational correlation function measures the probability that two bonds a distance $r$ apart are hexagonally oriented with respect to each other. It is expected that, although 2D lattices lack long range translational order, they do possess long range orientational order, so that $g_{6}(r)$ decays algebraically to a finite temperature-dependent level in the ordered state, whereas the decay goes over to exponential in the melt. Figure 7 shows the orientational correlation function $g_{6}(r)$ at several selected temperatures together with the guide-lines of characteristic $r^{-1 / 4}$ power law, expected at the hexatic-isotropic transition.

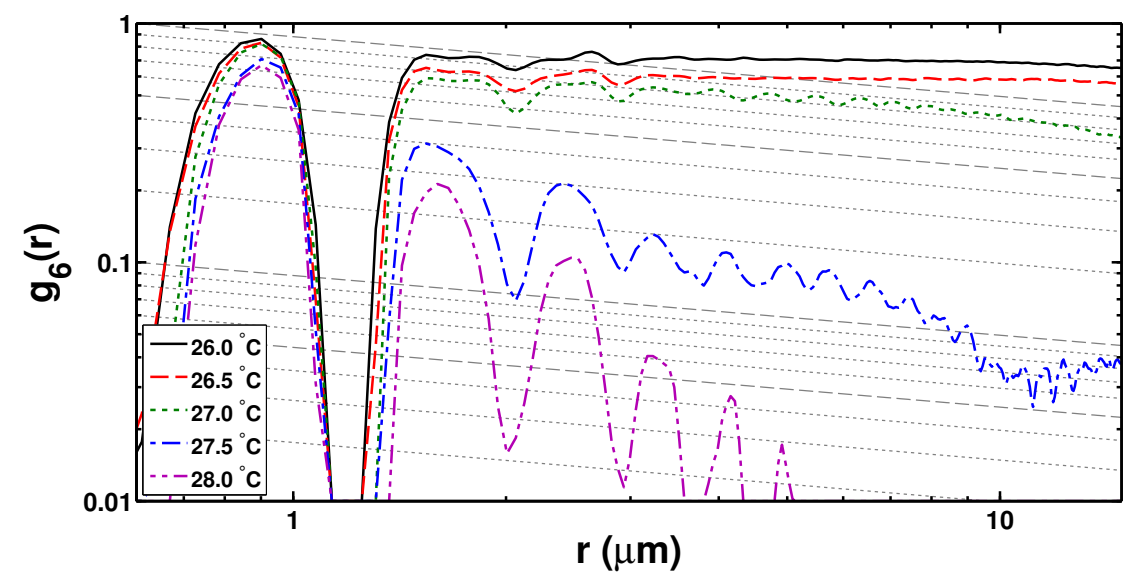

Figure 7. Bond-orientational correlation functions $g_{6}(r)$ of $2 \mathrm{D}$ colloidal structures at indicated temperatures. Slanted dashed lines are power laws $r^{-1 / 4}$, presented as guides to the eye.

The correlation functions $g_{6}(r)$ in figure 7 exhibit considerable oscillations due to local order analogous to, yet smaller than the corresponding oscillations of $g_{\mathrm{G}}(r)$ in figure 6 (a). Similarly to the case of $g_{\mathrm{G}}(r)$, we are only interested in the (smooth) behavior of the corresponding envelopes. Concentrating first on the lowest temperatures $26^{\circ} \mathrm{C}$ and $26.5^{\circ} \mathrm{C}$, where one expects the highest long-range orientational order, the correlation indeed stays almost constant with the distance, and thus indeed reveals a long-range orientational order. The decay is neither exponential, nor algebraic, although it is clearly slower than $r^{-1 / 4}$. At $27^{\circ} \mathrm{C}$, the dependence is rather algebraic and closely follows $r^{-1 / 4}$. At $27.5^{\circ} \mathrm{C}$ the decay is clearly faster than $r^{-1 / 4}$, whereas at $28.0^{\circ} \mathrm{C}$ it is still faster and close to exponential, indicating that melting is complete.

Comparing now the results of figure 6] and figure 7, they are qualitatively consistent with the KTHNY expectations. Firstly, the translational functions in figure 6 decay faster than the corresponding orientational ones in figure 7 at least at $T \leqslant 27.5^{\circ} \mathrm{C}$. It appears that the system enters the intermediate hexatic phase at a temperature around $27^{\circ} \mathrm{C}$, where the orientational function $g_{6}(r)$ exhibits a characteristic algebraic $r^{-1 / 4}$ behavior, whereas the translational function 
decay is close to $r^{-1 / 3}$, and the translational correlation length starts to drop. At $T \geqslant 27.5^{\circ} \mathrm{C}$, both $g_{6}(r)$ and $g_{\mathrm{G}}(r)$ decay rapidly, signaling the transition to isotropic state. Overall, it thus appears that the transition happens in a finite but narrow range around $27^{\circ} \mathrm{C}$.

Investigating colloidal systems with varying droplet size, we found that the $2 \mathrm{D}$ melting transition and the temperature range of intermediate phase depend on the size of glycerol droplets. In the case of smaller droplets of a diameter $2 R \simeq 1 \mu \mathrm{m}$, the transition occurs approximately at $27^{\circ} \mathrm{C}$. For larger droplets, the transition temperature increases, and for droplets of a diameter $2 R \simeq 2.5 \mu \mathrm{m}$ there was no melting observed within the nematic phase range of $5 \mathrm{CB}$ liquid crystal. Such difference is due to the fact that the NLC-mediated interactions, which stabilize the 2D lattice, are size-dependent and their magnitude increases with the droplet size. Thus, larger droplets are more strongly bounded, and thus their positions exhibit less thermal fluctuations. On the contrary, 2D lattices composed of smaller droplets stronger "feel" the changes in temperature.

\section{Lindemann parameter}

Lindemann condition [30] is a well established empirical melting criterion for 3D solids. In current interpretation, it states that the crystal melts when r.m.s. (root-mean-square) displacement of particles from their equilibrium positions due to thermal motion $\sqrt{\left\langle\Delta \mathbf{r}^{2}\right\rangle}$ reaches $15 \%$ of the lattice period $a$. Accordingly, one introduces the Lindemann parameter $L=\sqrt{\left\langle\Delta r^{2}\right\rangle} / a$, whose value is 0.15 at melting. It turns out that this criterion is also valid in higher dimensions, at least up to $D=50$ [31]. In $2 \mathrm{D}$ crystals, however, the situation is different: the particle deviations diverge at long times (for the same reason that $2 \mathrm{D}$ crystals do not possess long range order), so that the Lindemann parameter, as defined above, appears to have no meaning. The definition can however be easily adapted to a form suitable to $2 \mathrm{D}$ cases, either through the relative displacement of nearest neighbors $i$ and $i+1$ [14, 32], with the "modified" $L_{\mathrm{m}}=\sqrt{\left\langle\left(\Delta \mathbf{r}_{i}-\Delta \mathbf{r}_{i+1}\right)^{2}\right\rangle} / a$, or else by evaluating the displacements $\Delta \mathbf{r}$ in local coordinate systems of the particles [33]. In both cases, the diverging long-wavelength fluctuations are excluded, so that the result is finite and essentially equivalent to the usual definition. Numerically, however, $L_{\mathrm{m}}$ and $L$ are not identical - indeed, assuming uncorrelated motion of neighboring particles, as in the Einstein solid, then evidently $L_{\mathrm{m}}^{2}=2 L^{2}$. Furthermore, some authors use $L^{2}$ in place of $L$ and/or introduce additional numerical pre-factors into the definition. Due care has therefore to be taken when comparing data from the literature. In the following, we assume the classical definition of the Lindemann parameter, and accordingly reduce the literature data for comparisons, when needed.

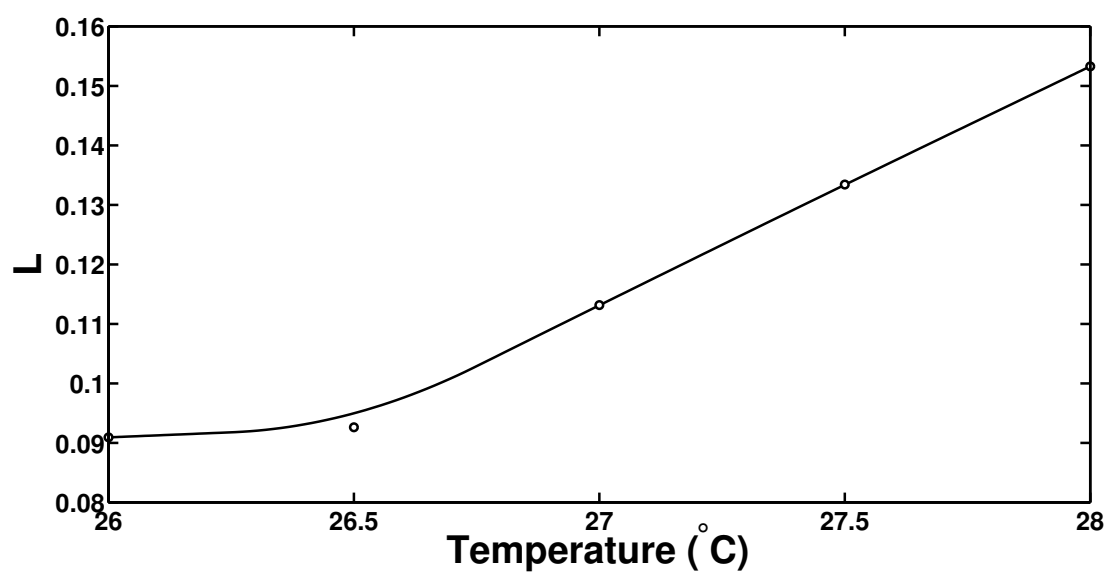

Figure 8. Temperature dependence of the Lindemann parameter, estimated from the standard deviation of the distribution of colloidal bond lengths.

In order to estimate $L$ in our case, we observe that in the case when $|\Delta \mathbf{r}| \ll a$ and assuming uncorrelated motion of neighboring particles, $L_{\mathrm{m}}^{2}$ essentially gives the bond length variance, 
$L_{\mathrm{m}}^{2} \approx\left\langle(|\mathbf{b}|-a)^{2}\right\rangle$, where $\mathbf{b}$ is the bond vector between the nearest neighbors $i$ and $i+1$. We therefore use r.m.s. bond length fluctuation to estimate the Lindemann parameter, $L=\sqrt{\left\langle(|\mathbf{b}|-a)^{2}\right\rangle} / \sqrt{2} a$. Evidently, the r.m.s. bond length fluctuation overestimates $L$, since it also reflects the static bondlength disorder, which is significant in the intermediate phase. Yet we believe that, since the dynamic fluctuations are relatively large at the transition, it gives an acceptable estimate. Temperature dependence of the so-determined Lindemann parameter is presented in figure 8 . One observes that $L=0.09$ in the crystal and starts to rise linearly with the temperature at $26.5^{\circ} \mathrm{C}$, i. e. where, according to the previous analyses, the system enters the intermediate phase. At melting, which occurs somewhat above $27^{\circ} \mathrm{C}$, it reaches the value $L \approx 0.12$ or 0.13 , which is thus rather close to the $3 \mathrm{D}$ condition of $L=0.15$.

It has been suggested in the literature [14, 15, 32] that $L_{\mathrm{c}}=0.13$ is the critical value, at which $2 \mathrm{D}$ solid melts (the quoted papers actually used $L_{\mathrm{m}}^{2}$ as the modified Lindemann parameter, from which $L=\sqrt{L_{\mathrm{m}}^{2} / 2}$ ). We confirm that our system melts at, or close to this value, which thus seems to be universal, although somewhat different estimates of $L_{\mathrm{c}}$ were also suggested, such as $L_{\mathrm{c}} \sim 0.1$ in an MD simulation work [33]. Overall, it appears that $L_{\mathrm{c}}$ does exist in $2 \mathrm{D}$, and is close to the corresponding $3 \mathrm{D}$ value of 0.15 . We note that the existence of the Lindemann criterion in $2 \mathrm{D}$, although not explicitly discussed in the KTHNY theory, is closely related to the critical elastic moduli of the theory, e. g., to Young's modulus $K$, whose value at the solid-hexatic transition is such that $K a^{2} / k_{\mathrm{B}} T=16 \pi$ [10, 34]. Approximating the colloidal system with the 2D Einstein solid, where each particle vibrates in a harmonic potential $U=k r^{2} / 2$ created by its surrounding, it follows from mean-field-like considerations that the modulus $K$ is proportional to the corresponding spring constant $k$. The latter, however, is directly related to the Lindemann factor. Indeed, mean square displacement $\left\langle r^{2}\right\rangle$ of a $2 \mathrm{D}$ harmonic oscillator is given by its thermal energy $k_{\mathrm{B}} T$ such that $\left\langle r^{2}\right\rangle=2 k_{\mathrm{B}} T / k$ [35], which, introducing the Lindemann parameter, leads to $k a^{2} / k_{\mathrm{B}} T=2 / L^{2}$, so that the elastic constant (and thus the modulus) is related to $L$. Consequently, the existence of a critical elastic modulus implies the existence of $L_{\mathrm{c}}$.

Finally, we try to estimate the depth of the potential wells of lattice sites. We approximate the potential, which is periodic with the lattice period $a$, with the trigonometric function $U(x)=$ $U_{0} \sin ^{2} \pi x / a$, so that the corresponding elastic constant is $k=2 \pi^{2} U_{0} / a^{2}$. From the reasoning of the previous paragraph it then follows that $U_{0}=k_{\mathrm{B}} T / \pi^{2} L^{2}$. Thus, the depth of the potential wells decreases from $U_{0} \simeq 12 k_{\mathrm{B}} T$ at $26.0^{\circ} \mathrm{C}$ to $U_{0} \simeq 6 k_{\mathrm{B}} T$ at $28.0^{\circ} \mathrm{C}$.

\section{Conclusions}

In conclusion, we have shown that melting of a two-dimensional colloidal system, formed by glycerol droplets at the free surface of a nematic liquid crystalline layer, occurs qualitatively and even semi-quantitatively in accordance with the Kosterlitz-Thouless-Halperin-Nelson-Young (KTHNY) theory. In particular, melting is obviously a continuous transition through an intermediate hexatic phase, characterized by quick rise of the number of structural defects. However, the temperature range of the intermediate phase is rather narrow, perhaps $\lesssim 1^{\circ} \mathrm{C}$. Furthermore, decay of the correlation functions with distance can only roughly be approximated with the characteristic critical power laws and exponential decays in different phases, suggesting that the details of the transition scenario do not fully comply with the KTHNY expectations, and reflect peculiarities of the interparticle interaction.

\section{Acknowledgements}

The work was supported by National Academy of Sciences of Ukraine AS of Ukraine via Grants VC-134 and \#5M/09-16; and Fundamental Research State Fund Project UU24/018. 


\section{References}

1. Bénard H., Rev. Gen. Sci. Pure Appl., 1900, 11, 1261; 1900, 11, 1309; Ann. Chim. Phys., 1901, 23, 62.

2. Murray C.A., Grier D.G., Annu. Rev. Phys. Chem., 1996, 47, 421.

3. Melzer A., Homann A., Piel A., Phys. Rev. E, 1996, 53, 2757.

4. Ziman J.M. Models of Disorder: The Theoretical Physics of Homogeneously Disordered Systems. Cambridge Univ. Press, London, 1979.

5. Peierls R.E., Ann. Inst. Henri Poincaré, 1935, 5, 177-222.

6. Mermin N.D., Phys. Rev., 1968, 176, 250.

7. Kosterlitz J.M., Thouless D.J., J. Phys. C, 1973, 6, 1181.

8. Halperin B.I., Nelson D.R., Phys. Rev. Lett., 1978, 41, 121; Nelson D.R., Halperin B.I., Phys. Rev. B, 1979, 19, 2457.

9. Young A.P., Phys. Rev. B, 1979, 19, 1855.

10. Strandburg K.J., Rev. Mod. Phys., 1988, 60, 161.

11. Bond-Orientational Order in Condensed Matter Systems, edited by Strandburg K.J. Springer, New York, 1992.

12. Gasser U., J. Phys.: Condens. Matter, 2009, 21, 203101.

13. Chui S.T., Phys. Rev. B, 1983, 28, 178.

14. Zahn K., Lenke R., Maret G., Phys. Rev. Lett., 1999, 82, 2721.

15. Zahn K., Maret G., Phys. Rev. Lett., 2000, 85, 3656.

16. Marcus A.H., Rice S.A., Phys. Rev. E, 1997, 55, 637.

17. Murray C.H., Van Winkle D.H., Phys. Rev. Lett., 1987, 58, 1200.

18. Nosenko V., Zhdanov S.K., Ivlev A.V., Knapek C.A., Morfill G.E., Phys. Rev. Lett., 2009, 103, 015001.

19. Fortov V.E., Ivlev A.V., Khrapak S.A., Khrapak A.G., Morfill G.E., Phys. Rep., 2005, 421, 1.

20. Zheng X.H., Grieve R., Phys. Rev. B, 2006, 73, 064205.

21. Nazarenko V.G., Nych A.B., Lev B.I., Phys. Rev. Lett., 2001, 87, 075504.

22. Smalyukh I.I., Chernyshuk S., Lev B.I., Nych A.B., Ognysta U., Nazarenko V.G., Lavrentovich O.D., Phys. Rev. Lett., 2004, 93, 117801.

23. Nych A.B., Ognysta U.M., Pergamenshchik V.M., Lev B.I., Nazarenko V.G., Musevic I., Skarabot M., Lavrentovich O.D., Phys. Rev. Lett., 2007, 98, 057801.

24. Crocker J.C., Grier D.G., J. Colloid Interface Sci., 1996, 179, 298.

25. Ognysta U.M., Nych A.B., Nazarenko V.G., Lev B.I., Ukr. J. Phys., 2007, 52, 633.

26. Hansen J.-P., Verlet L., Phys. Rev., 1969, 184, 151.

27. Ramakrishnan T.V., Yussouff M., Phys. Rev. B, 1979, 19, 2775.

28. Broughton J.Q., Gilmer G.H., Weeks J.D., Phys. Rev. B, 1982, 25, 4651.

29. Gonzales D.J., Gonzales L.E., Silbert M., Mol. Phys., 1991, 74, 613.

30. Lindemann F.A., Phys. Z., 1910, 11, 609.

31. Finken R., Schmidt M., Löwen H., Phys. Rev. E, 2001, 65, 016108.

32. Bedanov V.M., Gadiyak G.V., Phys. Lett A, 1985, 109, 289.

33. Zheng X.H., Earnshaw J.C., Europhys. Lett., 1998, 41, 635.

34. Keim P., Maret G., von Grünberg H.H., Phys. Rev. E, 2007, 75, 031402.

35. Chandrasekhar S., Rev. Mod. Phys., 1943, 15, 1. 


\title{
Плавлення двовимірної рідкокристалічної колоїдної структури
}

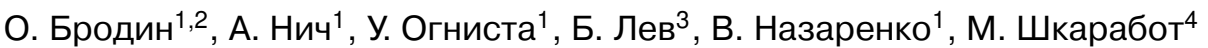 \\ І. Мушевич, \\ 1 Інститут фізики НАН України, Київ, Україна \\ 2 Національний технічний університет України “Київський політехнічний інститут”, Київ, Україна \\ 3 Інститут теоретичної фізики ім. М.М. Боголюбова НАН України, Київ, Україна \\ 4 Інститут Й. Стефана, Любляна, Словенія
}

\begin{abstract}
Використовуючи відеомікроскопію, ми дослідили плавлення двовимірної колоїдної системи, сформованої гліцериновими краплями на вільній поверхні шару рідкого кристала. Аналізуючи різні структурні кореляційні функції, ми показуємо, що плавлення відбувається через проміжну гексатичну фазу, як передбачено теорією Костерліца-Таулесса-Альперіна-Нельсона-Янга (КТАНЯ). Проте температурний діапазон проміжної фази $€$ дуже вузьким $\lesssim 1^{\circ} \mathrm{C}$ і характеристичний критичний степінь спадання кореляційних функцій не повністю встановлюється. Ми зробили висновок, що плавлення таких двовимірних систем якісно описується теорією КТАНЯ, хоча кількісні деталі сценарію переходу можуть частково залежати від деталей взаємодії між частинками.
\end{abstract}

Ключові слова: рідкі кристали, двовимірні колоїдні структури, фазові переходи в двовимірних структурах 